\title{
Influencia del nivel de deformación previa en el comportamiento a fatiga de metales dúctiles para embutición
}

\author{
C. GÓMEZ, J. L. NÚÑEZ, E. FULLOLA \\ Área de Mecánica y Nuevos Materiales. Instituto Tecnológico de Aragón (ITA) 50018 Zaragoza, España.
}

\begin{abstract}
En el presente trabajo se analiza la influencia en el comportamiento a fatiga que, sobre dos aceros con diferentes composiciones, ambos adecuados para la fabricación de productos mediante conformado metálico, tiene el nivel de deformación inicial y el nivel de deformación media bajo el que se realizan los ensayos. Los aceros analizados responden a la denominación DC05 (DIN EN 10130) y ZStE 260 (DIN EN 10268). En los dos casos se aceptan como válidas para describir el comportamiento de ambos materiales las expresiones propuestas por Ramberg-Osgood (1) para la curva tensión - deformación cíclica del material ( $\sigma-\varepsilon$ cíclica), así como las propuestas por Basquin (2), MansonCoffin (3), (4) para la expresión de la relación amplitud de deformación total - vida $(\varepsilon-\mathrm{N})$. La caracterización a fatiga de dichos materiales se realiza conforme a procedimientos de laboratorio previamente puestos en marcha en el Instituto Tecnológico de Aragón ((5) y (6)) usando como punto de partida las recomendaciones propuestas por la ASTM ((7), (8) y (9)). Debido a la geometría de las probetas analizadas (pequeño espesor, entorno a los $1.5 \mathrm{~mm}$.), los problemas más frecuentes encontrados han estado asociados al fenómeno de pandeo de las probetas bajo cargas de compresión, cuya solución se ha abordado mediante diversas técnicas experimentales y la fabricación de utillajes de ensayos adecuados (como se describe, entre otros, en (10)).
\end{abstract}

Palabras clave: Predeformación, fatiga, acero DC05, acero ZStE260, predicción de vida

\section{Influence of the previous strain level in the fatigue behaviour of ductile steel for deep drawing}

In the current work, the influence of the initial and medium strain under which fatigue tests are performed in the fatigue behaviour of two different steels for deep drawing is analysed. The two steels analysed has the commercial denomination DC05 (according to DIN EN 10130) and ZStE 260 (DIN EN 10268). In both cases, the expressions proposed by Ramberg-Osgood (1) for the cyclic stress-strain curve ( $\sigma-\varepsilon)$ as well as those proposed by Basquin (2) and Manson-Coffin (3),(4) for the relationship between strain amplitudes and life ( $\varepsilon$-N curve) are assumed as valid. The fatigue characterisation of both materials is made according to standard laboratory procedures, previously developed and setting up in the Instituto Tecnológico de Aragón ((5) and (6)), using for that as basis the recommendations proposed by ASTM ((7), (8) and (9)). The most usual problems found during the tests have been associated to buckling in the specimens under compression loads. Those problems, due to the geometry of the specimens tested (small thickness, about $1.5 \mathrm{~mm}$.), have been solved by using several experimental techniques and by manufacturing specific tests tooling (as is described in (10)).

Keywords:prestrain, material fatigue, DC05 steel material, ZStE260 steel material, fatigue life prediction

\section{INTRODUCCIÓN}

Los componentes de la industria actual, están generalmente sujetos a deformaciones previas debido a los procesos de fabricación en los que se ven envueltos, o a operaciones posteriores (ya sean intencionadas o no) sobre el producto terminado. Todas estas predeformaciones pueden ser potencialmente peligrosas y reducir considerablemente la vida a fatiga del componente o incluso beneficiosas si generan tensiones residuales opuestas a las cargas de servicio. Para una adecuada estimación de la vida a fatiga en estos componentes, tanto el daño como los efectos beneficiosos de estas predeformaciones deberían ser considerados por los modelos de predicción de vida a fatiga.

Para ello se ha analizado el efecto de la deformación inicial sobre dos materiales (DC05 y ZStE260) comúnmente empleados para la fabricación de componentes por conformado metálico. El comportamiento a fatiga se ha determinado mediante ensayos de amplitud de deformación constante sobre probetas sin predeformar y con una predeformación en tracción del 15\%, con el fin de establecer un análisis comparativo. Adicionalmente, en el caso del ZStE260, se ha analizado la influencia de la deformación media realizando los ensayos a $R_{\varepsilon}=0$ y $R_{\varepsilon}=-1$

\section{DETALLES DEL ENSAYO}

\subsection{Material y probetas}

Los materiales de partida para la realización de los ensayos han sido el DC05 (acero al carbono) y el ZStE260 (acero microaleado) cuyas composiciones químicas aparecen en la tabla I. Para la obtención de muestras predeformadas y sin predeformar, se ha partido de bandas de material de $50 \mathrm{~mm}$ de anchura y $600 \mathrm{~mm}$ de longitud, las cuales fueron troqueladas de planchas de chapa en bruto. Estas bandas fueron cortadas al menos a $100 \mathrm{~mm}$ de distancia de los extremos de las planchas, para evitar posibles efectos de borde por formación de ondas o por diferentes microestructuras causadas por gradientes de enfriamiento.

TABLA I. COMPOSICIÓN QUÍMICA

\begin{tabular}{|c|c|c|c|c|c|c|c|c|}
\hline Material & $\begin{array}{c}\mathrm{C} \\
(\% \text { max })\end{array}$ & $\begin{array}{c}\text { Mn } \\
(\% \text { max })\end{array}$ & $\begin{array}{c}\mathbf{P} \\
(\% \text { max })\end{array}$ & $\begin{array}{c}\mathbf{S} \\
(\% \text { max })\end{array}$ & $\begin{array}{c}\mathbf{S i} \\
(\% \text { max })\end{array}$ & $\begin{array}{c}\mathbf{A l} \\
(\% \text { max })\end{array}$ & $\begin{array}{c}\mathbf{N b} \\
(\% \text { max })\end{array}$ & $\begin{array}{c}\mathrm{Ti} \\
(\% \text { max })\end{array}$ \\
\hline DC05 & 0.06 & 0.35 & 0.025 & 0.025 & 0.10 & & & \\
\hline ZstE260 & 0.06 & 0.50 & 0.60 & 0.030 & & 0.015 & 0.09 & 0.22 \\
\hline
\end{tabular}

La predeformación uniaxial $\left(\varepsilon_{2}=-1 / 2 \varepsilon_{1}\right)$ se ha llevado a cabo sobre las bandas una vez troqueladas, mediante una máquina de tracción 
uniaxial, hasta alcanzar deformaciones plásticas del 15\%. Después de esto, las bandas han sido sometidas a un recocido durante 45 minutos a una temperatura de $200^{\circ} \mathrm{C}$.

Las probetas de fatiga han sido mecanizadas de las bandas predeformadas, utilizando un equipo de electroerosión. Este procedimiento cubre completamente los requerimientos en cuanto a perpendicularidad se refiere, además de tener una menor influencia sobre los bordes de las probetas, comparado con un mecanizado convencional. Las probetas han sido extraídas transversalmente $\left(\mathrm{rd}=90^{\circ}\right)$ a la dirección de enrrollamiento de las bandas de chapa. El espesor de las probetas varía entre $1.53 \mathrm{~mm}$ para el material ZstE 260 y $1.20 \mathrm{~mm}$ para el material DC05 (figura 1)

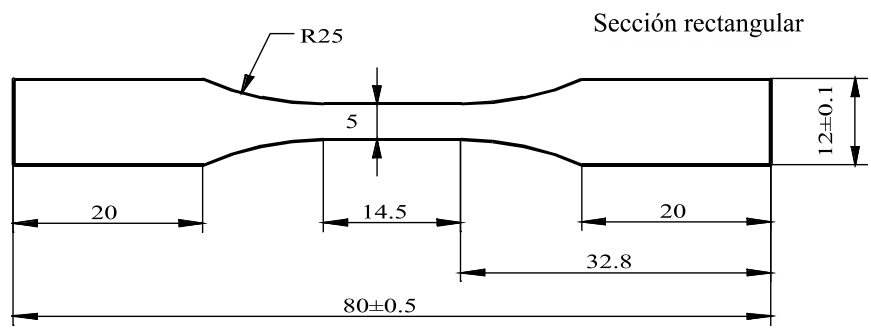

Figura 1. Probeta de ensayo. Dimensiones en mm.

\subsection{Equipamiento y condiciones de ensayo:}

El equipamiento de ensayo, empleado tanto para los ensayos estáticos como los cíclicos, ha consistido en una máquina de ensayos de alta frecuencia con un servocilindro hidráulico de $25 \mathrm{kN}$ (figura 2).

La deformación ha sido controlada mediante un extensómetro acoplado a los bordes de las probetas, con una longitud inicial entre marcas $\mathrm{L}_{0}=10 \mathrm{~mm}$. Las cargas de ensayo han sido generadas por un software de control, capaz de almacenar también los datos de ensayo resultantes. Todas las muestras han sido ensayadas a temperatura ambiente, bajo un rango de amplitudes de deformación constantes, aplicadas en la dirección longitudinal de las probetas.

Para la obtención de datos de partida que permitan la elaboración de un plan de ensayos optimizado, se han determinado las curvas monotónicas de los materiales (tensión real frente a deformación real), las cuales permiten la obtención tanto del límite como del módulo elástico de los mismos (ver resultados de los ensayos en tabla II). Las amplitudes de deformación empleadas para los ensayos de fatiga están incluidas en la zona elastoplástica de dichas curvas y la máxima amplitud de deformación se ha seleccionado de tal modo que no se alcance el $80 \%$ de la carga teórica de pandeo.

\section{TABLA II. RESULTADOS CURVAS MONOTÓNICAS}

\begin{tabular}{|l|c|c|c|c|}
\hline Material & $\mathbf{A}_{\mathbf{0}}(\mathbf{m m} 2)$ & $\mathbf{F}_{\mathrm{p} 0.2}(\mathbf{N})$ & $\mathbf{E}(\mathbf{G P a})$ & $\sigma_{\mathrm{p} 0.2}(\mathbf{M P a})$ \\
\hline DC05 (0\% predeformación) & 7.69 & 1444 & 211.8 & 187.8 \\
\hline DC05 (15\% predeformación) & 7.26 & 2477 & 193.5 & 341 \\
\hline ZStE260 (0\% predeformación) & 6.48 & 2074 & 202.33 & 320 \\
\hline ZStE260 (15\% predeformación) & 66.06 & 2866 & 202.39 & 473 \\
\hline
\end{tabular}

$\mathrm{A}_{0}=$ Área inicial; $\mathrm{F}_{\mathrm{pO2}}=$ Fuerza en el límite elástico; $\mathrm{E}=$ Módulo elástico o de Young; $\sigma_{\mathrm{p02}}=$ $\mathrm{F}_{\mathrm{p} 0.2} / \mathrm{A}_{0}$

La forma de la onda de la señal de deformación empleada es un seno. La forma de la onda así como la frecuencia de la misma se mantienen constantes durante todo el ensayo, realizándose éste a frecuencias de rango entre $3 \mathrm{~Hz}$ y $25 \mathrm{~Hz}$ (dependiendo de la amplitud de deformación), con el fin de conseguir un tiempo de ensayo no muy prolongado y asegurar un espectro de cargas y deformaciones tan exacto como fuera posible.

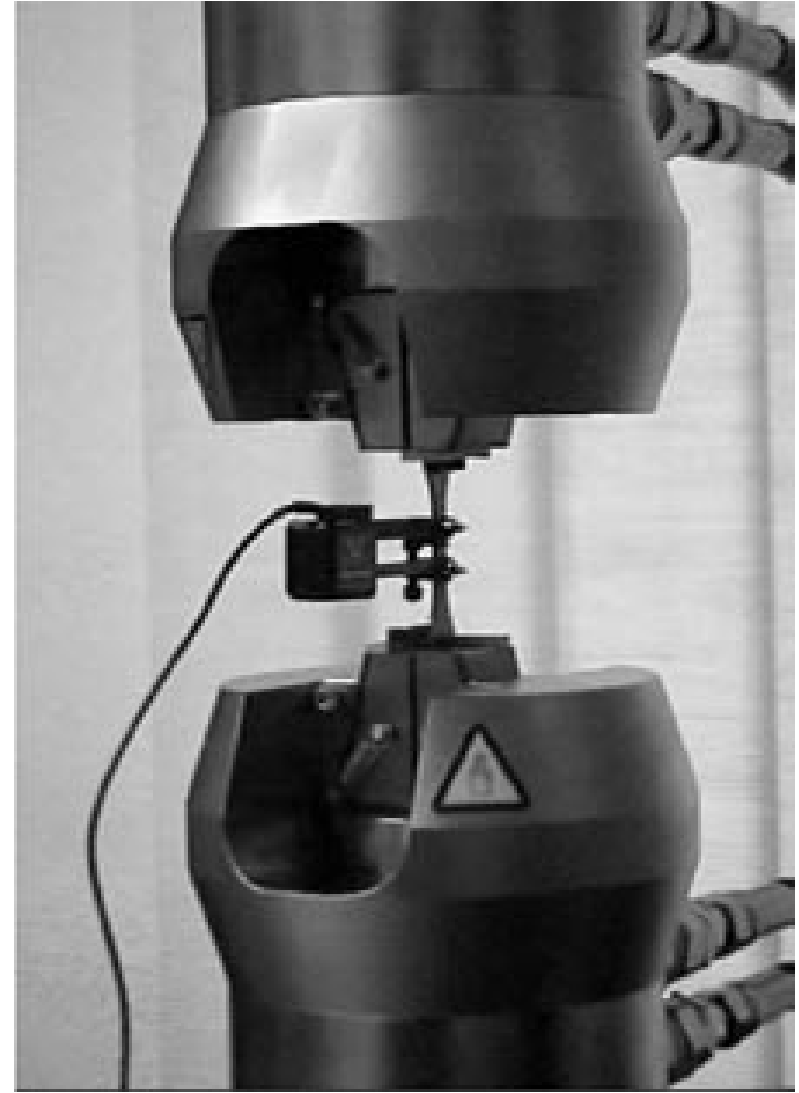

Figura 2. Probeta en ensayo

La parada del ensayo se realiza cuando se alcanza un número de ciclos predeterminado de $2 \times 10^{6}$ (criterio de "run out") o cuando la diferencia de amplitud de carga entre un ciclo y el anterior alcanza una variación del 50\% (pérdida de rigidez por aparición de grieta).

\section{PLAN DE ENSAYOS Y RESULTADOS:}

Para cada material se ha realizado un plan de ensayos obteniendo inicialmente la curva monotónica del material, citada anteriormente, y posteriormente su curva cíclica. Para ello se ha considerado, en la medida de lo posible, cuatro niveles de deformación y tres probetas por nivel, y se han determinado los valores de amplitud de deformación dentro de la zona elastoplástica del material. El plan de ensayos se detalla en la tabla III.

TABLA III. Plan INICIAL DE ENSAYOS

\begin{tabular}{|c|c|c|c|c|c|c|c|c|c|}
\hline Material & $\begin{array}{c}\text { Valor de } \\
\text { predeformación }\end{array}$ & $\begin{array}{c}\text { Ratio } \\
\text { deformación } \\
\left(\mathbf{R}_{\mathrm{\varepsilon}}\right)\end{array}$ & $\begin{array}{c}\mathbf{N}^{\mathrm{o}} \text { total } \\
\text { probetas }\end{array}$ & Niveles & \multicolumn{3}{|c|}{$\begin{array}{c}\text { Probetas } \\
\text { por nivel }\end{array}$} & \multicolumn{4}{|c|}{$\begin{array}{c}\text { Amplitud de } \\
\text { deformación \% }\end{array}$} \\
\hline DC05 & $0 \%$ & -1 & 12 & 4 & 3 & 0.09 & 0.10 & 0.12 & 0.13 \\
\hline DC05 & $15 \%$ uniaxial & -1 & 12 & 4 & 3 & 0.10 & 0.12 & 0.13 & 0.14 \\
\hline ZStE 260 & $0 \%$ & 0 & 12 & 4 & 3 & 0.11 & 0.12 & 0.14 & 0.15 \\
\hline ZStE 260 & $15 \%$ uniaxial & -1 & 12 & 4 & 3 & 0.13 & 0.15 & 0.17 & 0.21 \\
\hline
\end{tabular}

Durante la ejecución de los ensayos de fatiga se ha modificado ligeramente el plan de ensayos inicial previsto, en función de los resultados que se han ido obteniendo. Estos resultados se muestran en las tablas IV a VII siguientes: 
TABLA IV. DC05, 0\% PREDEFORMADO $\left(\mathrm{R}_{\mathrm{c}}=-1\right)$

\begin{tabular}{|c|c|c|c|c|c|}
\hline $\mathrm{N}^{\mathrm{o}}$ Probeta & $\varepsilon_{\mathrm{a}}(\%)$ & $\mathrm{A}_{0}(\mathrm{~mm} 2)$ & $\mathrm{F}_{\mathrm{a}}(\mathrm{N})$ & $\sigma a .(\mathrm{MPa})$ & $\mathrm{Nf}$ \\
\hline 2M000368.003 & 0.13 & 7.545 & 1075 & 142.48 & 139600 \\
\hline 2M000368.004 & 0.13 & 7.610 & 1091 & 143.36 & 131400 \\
\hline 2M000368.006 & 0.13 & 7.530 & 1083 & 143.82 & 155300 \\
\hline 2M000366.004 & 0.12 & 7.614 & 1147 & 150.64 & 130200 \\
\hline 2M000366.005 & 0.12 & 7.595 & 1149 & 151.28 & 180800 \\
\hline 2M000366.002 & 0.12 & 7.565 & 1120 & 148.05 & 172000 \\
\hline 2M000368.001 & 0.10 & 7.580 & 988 & 130.34 & 266135 \\
\hline 2M000368.005 & 0.10 & 7.617 & 1003 & 131.68 & 302000 \\
\hline 2M000367.006 & 0.09 & 7.227 & 1050 & 145.29 & 391000 \\
\hline 2M000367.002 & 0.09 & 7.495 & 1100 & 146.76 & 730000 \\
\hline 2M000368.002 & 0.09 & 7.654 & 1090 & 142.41 & 696840 \\
\hline
\end{tabular}

TABLA V. DC05, 15\% PREDEFORMADO $\left(\mathrm{R}_{\varepsilon}=-1\right)$

\begin{tabular}{|c|c|c|c|c|c|}
\hline $\mathrm{N}^{\mathrm{o}}$ Probeta & $\varepsilon_{\mathrm{a}}(\%)$ & $\mathrm{A}_{0}(\mathrm{~mm} 2)$ & $\mathrm{F}_{\mathrm{a}}(\mathrm{N})$ & $\sigma a .(\mathrm{MPa})$ & $\mathrm{N}_{\mathrm{f}}$ \\
\hline 2M000371.001 & 0.14 & 7.28 & 1273 & 174.89 & 112000 \\
\hline 2M000371.002 & 0.14 & 7.31 & 1277 & 174.74 & 160000 \\
\hline 2M000371.003 & 0.14 & 7.35 & 1281.5 & 174.32 & 182000 \\
\hline 2M000369.002 & 0.13 & 7.24 & 1263.5 & 174.44 & 262000 \\
\hline 2M000369.005 & 0.13 & 7.01 & 1256.5 & 179.3 & 370000 \\
\hline 2M000369.004 & 0.13 & 7.31 & 1186 & 162.14 & 352000 \\
\hline 2M000370.006 & 0.12 & 7.33 & 1240.5 & 169.25 & 483200 \\
\hline 2M000370.003 & 0.12 & 7.26 & 1243.5 & 171.34 & 357650 \\
\hline 2M000370.004 & 0.12 & 7.34 & 1232 & 167.81 & 430000 \\
\hline 2M000370.002 & 0.1 & 7.31 & 1193.7 & 163.34 & 737000 \\
\hline 2M000371.004 & 0.1 & 7.29 & 1151 & 157.81 & 1400000 \\
\hline 2M000371.005 & 0.1 & & & & $2000000\left(^{*}\right)$ \\
\hline
\end{tabular}

$\varepsilon_{\mathrm{a}}=$ Amplitud deformación $; \mathrm{A}_{0}=$ Area inicial $; \mathrm{F}_{\mathrm{a}}=$ Amplitud carga a $\mathrm{N}_{\mathrm{f}} / 2 ; \sigma \mathrm{a}=\mathrm{F}_{\mathrm{a}} / \mathrm{A}_{0} ; \mathrm{N}_{\mathrm{f}}$ $=$ Ciclos fallo;

(*) Run-out. No se incluyen el resto de los datos del ensayo.

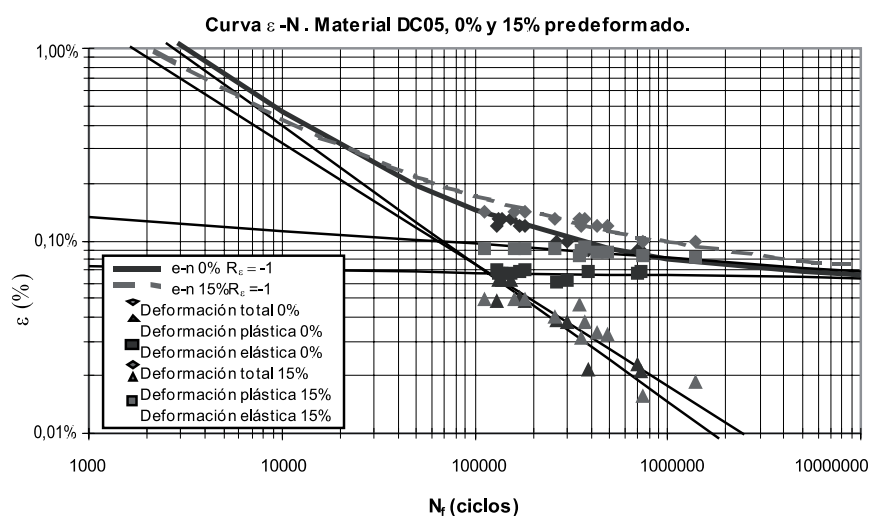

Figura 3. Curvas $\varepsilon-\mathrm{N}$ de Material DC05.

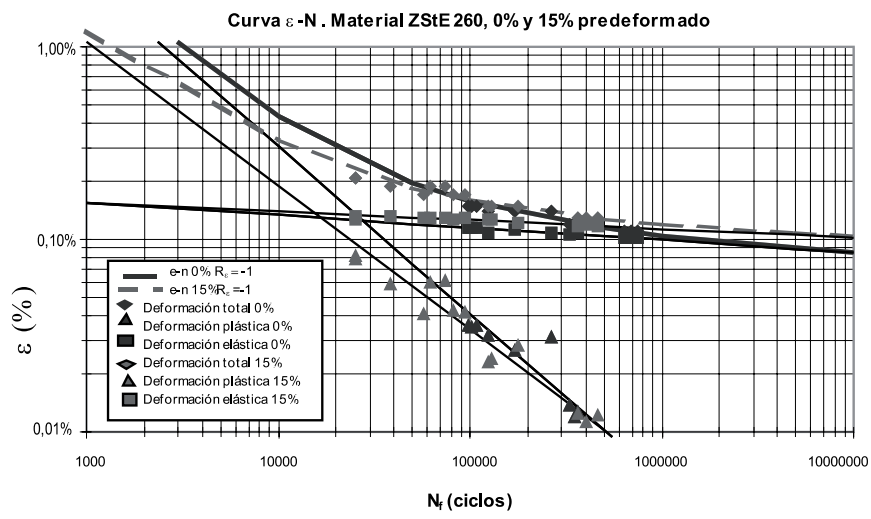

Figura 4. Curvas $\varepsilon$-N de Material ZStE 260.
TABLA VI. ZStE260, 0\% PREDEFORMADO $\left(\mathrm{R}_{\varepsilon}=0\right)$

\begin{tabular}{|c|c|c|c|c|c|}
\hline $\mathrm{N}^{\circ}$ Probeta & $\varepsilon_{\mathrm{a}}(\%)$ & $\mathrm{A}_{0}(\mathrm{~mm} 2)$ & $\mathrm{F}_{\mathrm{a}}(\mathrm{N})$ & $\sigma \mathrm{\sigma} .(\mathrm{MPa})$ & $\mathrm{N}_{\mathrm{f}}$ \\
\hline 2M000373.005 & 0.15 & 6.346 & 1470 & 231.640 & 109000 \\
\hline 2M000373.001 & 0.15 & 6.454 & 1493 & 231.330 & 98400 \\
\hline 2M000374.006 & 0.15 & 6.350 & 1482 & 233.386 & 102000 \\
\hline 2M000373.002 & 0.14 & 6.363 & 1462 & 229.770 & 170500 \\
\hline 2M000373.003 & 0.14 & 6.438 & 1420 & 220.565 & 265500 \\
\hline 2M000372.004 & 0.14 & 6.325 & 1383.5 & 218.735 & 123500 \\
\hline 2M000372.002 & 0.12 & 6.476 & 1393 & 215.100 & 330500 \\
\hline 2M000374.001 & 0.12 & 6.350 & 1387 & 218.425 & 354000 \\
\hline 2M000374.002 & 0.12 & 6.396 & 1390 & 217.323 & 368300 \\
\hline 2M000373.004 & 0.11 & 6.388 & 1323 & 207.110 & 693500 \\
\hline 2M000373.006 & 0.11 & 6.379 & 1320 & 206.930 & 756000 \\
\hline 2M000374.003 & 0.11 & 6.438 & 1317 & 204.567 & 637000 \\
\hline
\end{tabular}

TABLA VII. ZStE260, 15\% PREDEFORMADO $\left(\mathrm{R}_{\varepsilon}=-1\right)$

\begin{tabular}{|c|c|c|c|c|c|}
\hline $\mathrm{N}^{\circ}$ Probeta & $\varepsilon_{a}(\%)$ & $\mathrm{A}_{0}(\mathrm{~mm} 2)$ & $\mathrm{F}_{a}(\mathrm{~N})$ & $\sigma a .(\mathrm{MPa})$ & $\mathrm{N}_{\mathrm{f}}$ \\
\hline 2M000377.003 & 0.21 & 6.012 & 1559 & 259.315 & 25200 \\
\hline 2M000377.005 & 0.21 & 5.892 & 1561 & 264.936 & 25500 \\
\hline 2M000375.003 & 0.19 & 6.030 & 1587 & 263.184 & 61500 \\
\hline 2M000376.003 & 0.19 & 6.066 & 1588 & 261.787 & 74000 \\
\hline 2M000377.004 & 0.19 & 5.982 & 1590 & 265.810 & 38200 \\
\hline 2M000376.002 & 0.17 & 6.070 & 1579 & 260.132 & 95000 \\
\hline 2M000377.001 & 0.17 & 6.036 & 1561 & 258.615 & 82000 \\
\hline 2M000377.006 & 0.17 & 5.8617 & 1528 & 260.675 & 57200 \\
\hline 2M000376.004 & 0.15 & 6.086 & 1501 & 246.632 & 179800 \\
\hline 2M000376.006 & 0.15 & 6.024 & 1535 & 254.814 & 129000 \\
\hline 2M000377.002 & 0.15 & 5.900 & 1517 & 257.118 & 125000 \\
\hline 2M000375.001 & 0.13 & 6.086 & 1451 & 238.416 & 464500 \\
\hline 2M000375.004 & 0.13 & 6.002 & 1442 & 240.253 & 406000 \\
\hline 2M000376.005 & 0.13 & 6.000 & 1427 & 237.833 & 368000 \\
\hline
\end{tabular}

El modelo matemático utilizado para describir el comportamiento en ambos materiales es el propuesto por Ramberg-Osgood (1) para la curva tensión-deformación cíclica ( $\sigma-\varepsilon$ cíclica):

$$
\varepsilon_{\mathrm{a}}=\varepsilon_{\mathrm{a}, \mathrm{e}}+\varepsilon_{\mathrm{a}, \mathrm{p}} \quad \varepsilon_{\mathrm{a}, \mathrm{e}}=\frac{\sigma \mathrm{a}}{\mathrm{E}} \quad ; \quad \varepsilon_{\mathrm{a}, \mathrm{p}}=\left(\frac{\sigma_{\mathrm{a}}}{\mathrm{K}^{\prime}}\right)^{\frac{1}{\mathrm{n}^{\prime}}}
$$

y las expresiones propuestas por Basquin (2), Manson-Coffin (3) y (4) para la relación amplitud de deformación total-vida $\left(\varepsilon-\mathrm{N}_{\mathrm{f}}\right)$ :

Los parámetros cíclicos característicos para cada material se muestran en la tabla VIII:

$$
\varepsilon_{\mathrm{a}}=\varepsilon_{\mathrm{a}, \mathrm{e}}+\varepsilon_{\mathrm{a}, \mathrm{p}} \quad \varepsilon_{\mathrm{a}, \mathrm{e}}=\frac{\sigma \mathrm{f}^{\prime}}{\mathrm{E}} \cdot\left(2 \mathrm{~N}_{\mathrm{f}}\right)^{\mathrm{b}} \quad ; \varepsilon_{\mathrm{a}, \mathrm{p}}=\varepsilon_{\mathrm{f}}{ }^{\prime}\left(2 \mathrm{~N}_{\mathrm{f}}\right) \mathrm{c}
$$

Tabla VIII. Parámetros de Ramberg-Osgood y BasQuin, MansonCOFFIN

\begin{tabular}{|l|c|c|c|c|c|c|}
\hline \multicolumn{1}{|c|}{ Material } & $\mathbf{K}^{\prime}$ & $\mathbf{n}^{\prime}$ & $\sigma_{\mathbf{f}}^{\prime}$ & $\mathbf{b}$ & $\varepsilon_{\mathbf{f}}^{\prime}$ & $\mathbf{c}$ \\
\hline DC05, 0\% predeformado & $152.24 \mathrm{MPa}$ & 0.0079 & $172.3 \mathrm{MPa}$ & -0.0142 & 4.7123 & -0.7155 \\
\hline DC05, 15\% predeformado & $286.945 \mathrm{MPa}$ & 0.0658 & $448.370 \mathrm{MPa}$ & -0.0722 & 1.6305 & -0.6291 \\
\hline ZStE 260, 0\% predeformado & $386.15 \mathrm{MPa}$ & 0.0656 & $519.15 \mathrm{MPa}$ & -0.0655 & 15.7766 & -0.8647 \\
\hline ZStE 260, 15\% predeformado & $387.207 \mathrm{MPa}$ & 0.0524 & $442.207 \mathrm{MPa}$ & -0.04517 & 3.127 & -0.7479 \\
\hline
\end{tabular}

En las figuras 3 y 4 se muestran las curvas amplitud de deformación-vida $\left(\varepsilon-\mathrm{N}_{\mathrm{f}}\right)$ obtenidas para cada material respectivamente (DC05 y ZstE 260), comparando niveles de predeformación inicial del material. La figura 5 representa las curvas tensión-deformación cíclica ( $\sigma-\varepsilon$ cíclica) de cada material respectivamente (DC05 y ZstE 260) para los distintos niveles de predeformación. 


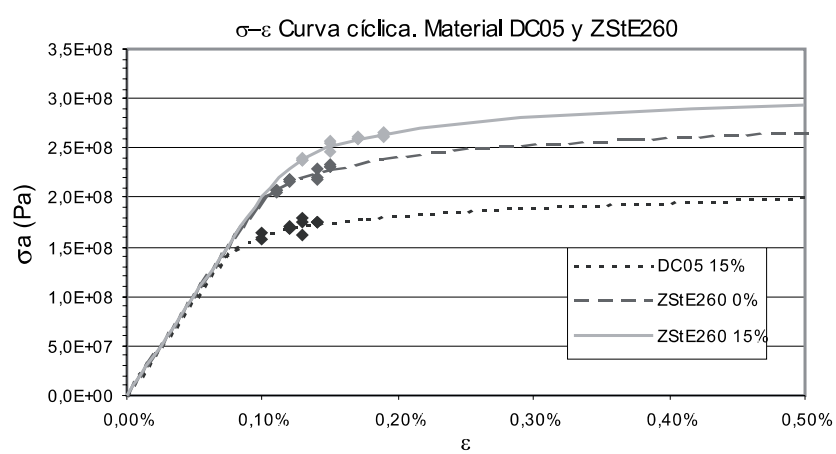

Figura 5.

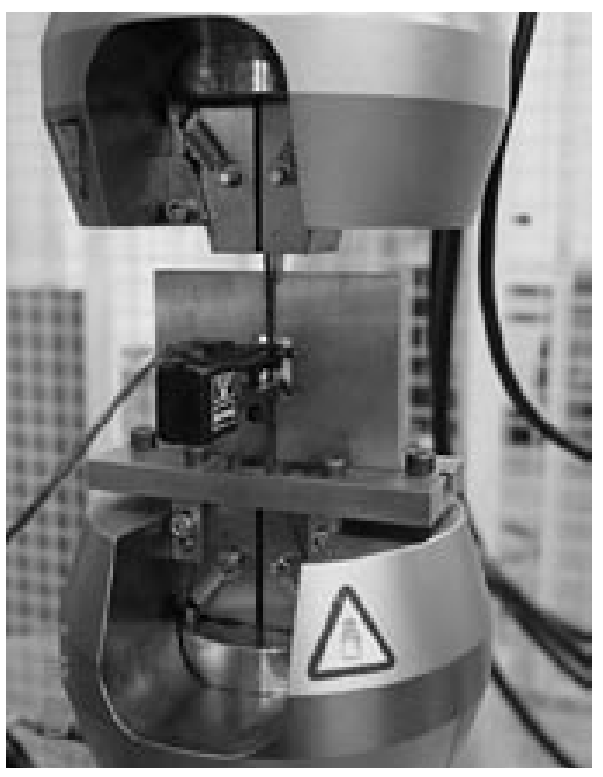

Figura 6. Dispositivo "anti-pandeo"

\section{ANÁLISIS Y CONCLUSIONES}

Como puede observarse de los resultados obtenidos para el acero DC05 (figura 3), el comportamiento a fatiga presentado por dicho material bajo condiciones de predeformación y sin ellas, difiere entorno al $20 \%$ en los niveles de deformación necesarios para alcanzar una vida de 1.000.000 de ciclos (mejor comportamiento a fatiga del material predeformado). No obstante, esta diferencia de comportamiento tiende a reducirse conforme se somete al material a amplitudes de deformación más elevadas, en los que los niveles de amplitud de deformación plástica (hasta del $0.065 \%$ ) son del mismo orden que los de deformación elástica (hasta el $0.09 \%$ ). De hecho, para niveles de deformación plástica elevados, las curvas deformación - vida del material predeformado y sin predeformar tienden a cruzarse, presentando un mejor comportamiento a fatiga el material no predeformado. Es importante remarcar que el rango en el que se ha trabajado con este material está comprendido entre los 100.000 y los 1.500 .000 ciclos, por lo que extrapolar las conclusiones obtenidas fuera de dicho rango, puede no ser muy adecuado. Los intentos que se realizaron para ensayar probetas de este material a niveles de deformación más elevados (por encima del $0.14 \%$ de amplitud de deformaciones) fracasaron, debido a la aparición de fenómenos de inestabilidades estructurales a compresión (pandeos).

Para la resolución de dicho problema, aparecido por el pequeño espesor y elevada esbeltez de las probetas utilizas (entorno a $1.5 \mathrm{~mm}$ ), se plantearon dos técnicas alternativas. La primera de ellas consistía en ensayar el material tan solo en tracción (es decir, con un ratio de deformación, $R_{\varepsilon^{\prime}}$ de 0). Esta técnica presentaba el problema de introducir una nueva variable de incertidumbre en la caracterización del material (el efecto de la deformación media impuesta) y fue utilizada en el acero ZstE260 no predeformado (ya que, al presentar un menor límite elástico que el predeformado, era susceptible de sufrir problemas de inestabilidades estructurales a compresión). La segunda de las técnicas consistía en la realización de un dispositivo "anti-pandeo", cuyas ideas generales fueron extraídas de (10) (ver figura 6). Este dispositivo ha sido ya fabricado y probado con éxito sobre probetas de prueba de acero St52 y será utilizado para, en los próximos meses, extender el rango de los resultados obtenidos sobre el material DC05 hasta el orden de las decenas de miles de ciclos (amplitudes de deformación de hasta $0.20 \%$ aproximadamente).

Conforme a lo anteriormente comentado, los resultados presentados para el material ZstE260 muestran dos influencias mezcladas: el nivel de predeformación al que se ha sometido el material inicialmente y el nivel medio de carga con el que se realiza el ensayo. Como puede observarse, y gracias tanto al mayor límite elástico del material (en su aspecto predeformado) como a la utilización de un ratio de deformaciones igual a 0 (en el caso del material no predeformado), los rangos de ciclos de vida hasta el fallo obtenidos en este caso se han encontrado entre los 30.000 y los 800.000 ciclos (que corresponden con amplitudes de deformación elásticas máximas de hasta $0.14 \%$ y plásticas de hasta $0.065 \%$ ). Como puede observarse en la figura 4 , la diferencia entre la amplitud de deformaciones que producen vidas de entorno a 1.000.000 de ciclos es menor del $10 \%$ para ambas condiciones del material (con un mejor comportamiento para el material predeformado y ratio de deformaciones -1). De nuevo, y ahora de manera más acusada, se produce un cambio en este comportamiento cuando las amplitudes de deformación a las que se somete al material hacen que éste se encuentre en el rango de fatiga a bajos ciclos ("low cycle fatigue", inferior a 50.000 ciclos).

No obstante, con estos resultados es posible concluir que, para el material microaleado, la influencia tanto del nivel de predeformación del mismo como del nivel medio de carga al que se realiza el ensayo son pequeñas en su vida a fatiga, siendo la variable dominante en este proceso de degradación la amplitud de deformaciones a la que se le somete.

De la comparación de valores de vida obtenidos para ambos materiales (DC05 y ZstE 260, ambos predeformados y con un ratio de deformación -1), es posible concluir el mejor comportamiento a fatiga del acero microaleado: soporta amplitudes de deformación mayores en un $20 \%$ para niveles de vida entorno a 1.000 .000 de ciclos (fatiga a altos ciclos) y de aproximadamente un $6 \%$ para niveles de vida de entorno a 100.000 ciclos (límite superior de la fatiga a bajos ciclos). Este mejor comportamiento puede también observarse en la figura 5, curvas cíclicas del material, en donde el acero ZstE 260 presenta un mayor límite elástico que el acero DC05.

\section{AGRADECIMIENTOS}

Este artículo se enmarca dentro de la línea de investigación en materiales metálicos desarrollada por el Área de Mecánica y Nuevos Materiales del ITA, que ha sido financiada parcialmente por el MCyT y por fondos FEDER, dentro del proyecto "Diseño a fatiga mediante modelos estadísticos de daño acumulado y elementos finitos probabilistas (2FD97-2423 TPP)".

\section{BIBLIOGRAFÍA}

1. W. Rambert \& W.R. Osgood, "Description of stress-strain curves by three parameters", NACA Tech. Note

2. O.H. Basquin, "The exponential law of endurance tests", Proc. ASTM, Vol. 10, Part II, 1910

3. J. F. Tavernelli \& L.F. Coffin Jr. "Experimental support for generalized equation predicting low cycle fatigue". Trans. ASME, J.Basic. Eng., Vol. 84, No 4, 1962

4. S.S. Manson,. "Discussion of reference 23", Trans. ASME J. Basic Eng., Vol. 84, No 4.S. Man 1962

5. Procedimiento ITA, "Procedimiento de ensayo para la determinación de la curva 5rocedimiento ITA, "Procedimiento de
de fatiga $\varepsilon$-N de materiales metálicos".

6. Procedimiento ITA, "Procedimiento para el análisis de las curvas de fatiga tensiónvida (S-N) y deformación - vida ( $\varepsilon$-N) de materiales metálicos".

7. ASTM E206-72.- "Fatigue testing and the statistical analysis of fatigue data".

8. ASTM E468-82.- "Presentation of constant amplitude fatigue test results for metallic materials".

9. ASTM E513-74.- "Constant-amplitude, low-cycle fatigue testing".

10. NACA 931.- "Guides for preventing buckling in axial fatigue tests on thin-sheet metal specimens". 